\title{
Assessment of Potential Cardiotoxic Side Effects of Mitoxantrone in Patients with Multiple Sclerosis
}

\author{
Vera Carina Zingler ${ }^{\mathrm{a}}$ Michael Näbauer ${ }^{\mathrm{b}}$ Klaus Jahn ${ }^{\mathrm{a}}$ Ariane Groß ${ }^{\mathrm{a}}$ \\ Reinhard Hohlfeld ${ }^{c}$ Thomas Brandt ${ }^{\mathrm{a}}$ Michael Strupp ${ }^{\mathrm{a}}$ \\ Departments of a Neurology, ${ }^{b}$ Cardiology and ${ }^{c}$ Neuroimmunology, University of Munich, Munich, Germany
}

\section{Key Words}

Mitoxantrone - Multiple sclerosis · Cardiotoxicity ·

Echocardiogram $\cdot$ Cardiomyopathy $\cdot$ Ejection fraction

\begin{abstract}
Previous studies showed that mitoxantrone can reduce disability progression in patients with multiple sclerosis (MS). There is, however, concern that it may cause irreversible cardiomyopathy with reduced left ventricular (LV) ejection fraction (EF) and congestive heart failure. The aim of this prospective study was to investigate cardiac side effects of mitoxantrone by repetitive cardiac monitoring in MS patients. The treatment protocol called for ten courses of a combined mitoxantrone $\left(10 \mathrm{mg} / \mathrm{m}^{2}\right.$ body surface) and methylprednisolone therapy. Before each course, a transthoracic echocardiogram was performed to determine the LV end-diastolic diameter, the end-systolic diameter and the fractional shortening; the LV-EF was calculated. Seventy-three patients participated (32 males; age $48 \pm 12$ years, range $20-75$ years; 25 with primary progressive, 47 with secondary progressive and 1 with relapsing-remitting MS) who received at least four courses of mitoxantrone. Three of the $73 \mathrm{pa}-$ tients were excluded during the study $(2$ patients discontinued therapy; 1 patient with a previous history of ischemic heart disease developed atrial fibrillation after the second course of mitoxantrone). The mean cumulative dose of mitoxantrone was $114.0 \pm 33.8 \mathrm{mg}$. The mean
\end{abstract}

follow-up time was 23.4 months (range 10-57 months). So far, there has been no significant change in any of the determined parameters (end-diastolic diameter, endsystolic diameter, fractional shortening, EF) over time during all follow-up investigations. Mitoxantrone did not cause signs of congestive heart failure in any of the patients. Further cardiac monitoring is, however, needed to determine the safety of mitoxantrone after longer follow-up times and at higher cumulative doses.

Copyright $(2005$ S. Karger AG, Basel

\section{Introduction}

Previous studies showed that mitoxantrone, an anthracenedione with immunosuppressive effects, can reduce disability progression and clinical exacerbations in patients with multiple sclerosis (MS) [1-6]. Therefore, mitoxantrone is increasingly being used especially for secondary progressive (SP) MS and repeated courses. Despite this promising therapeutic effect, however, there is still much concern about the safety of this drug because of its potential cardiac side effects, which seem to increase with higher cumulative dosages $[6,7]$. The cardiotoxicity is most likely caused by the formation of iron chelates, which promote formation of reactive oxygen intermediates that lead to damage of myocardial tissue [8]. In the past, mitoxantrone-induced cardiac side effects were mainly observed in cancer patients (e.g. prostate or breast

\section{KARGER}

Fax +41613061234 E-Mail karger@karger.ch www.karger.com
(C) 2005 S. Karger AG, Basel 0014-3022/05/0541-0028\$22.00/0

Accessible online at: www.karger.com/ene
Vera C. Zingler, MD

University of Munich, Klinikum Grosshadern, Department of Neurology

Marchioninistrasse 15, DE-81377 Munich (Germany)

Tel. +49 897095 2585, Fax +49 8970955584

E-Mail vzingler@nro.med.uni-muenchen.de 
cancer) [9-12], who were treated antineoplastically with mitoxantrone. The predominant cardiac side effects were an asymptomatic decrease of the left ventricular (LV) ejection fraction (EF), irreversible cardiomyopathy and congestive heart failure (CHF). However, a potentiation of cardiotoxicity might be assumed in cancer patients, because mitoxantrone is often given in combination with other chemotherapy or radiation. Until now, only a few studies have investigated the safety of mitoxantrone when used as a single-agent therapy in MS. They were mainly based on clinical examination [13-16] or included only a limited number of patients with short follow-up times $[17,18]$. In this prospective study, the potential cardiotoxicity of mitoxantrone in MS patients was evaluated by repetitive cardiac monitoring before each treatment course. We present here the results of an interim analysis after the study had lasted 5 years.

\section{Methods}

\section{Study Population}

In this prospective study, conducted from 1998 to 2004, patients with a definite MS, initially according to the criteria of Poser et al. [19] and since 2001 according to McDonald et al. [20], and either a deteriorating primary progressive (PP), SP, or a severe relapsing-remitting (RR) MS were included. On entry into the study, the patients underwent a neurological examination and a quantitative assessment of disability using the expanded disability status scale (EDSS) of Kurtzke [21]. Only PP and SP patients who had worsened by at least 0.5 or more EDSS points during the preceding 12 months were enrolled. Before inclusion, all other disease-modifying therapies were discontinued. All patients had given their written informed consent to participate in the study.

\section{Treatment Protocol}

The treatment protocol called for ten courses of a combined mitoxantrone and methylprednisolone (MP) therapy. The first three courses were given at 3-month intervals. Course four was administered 4 months after the previous course; course five, 5 months after this, and courses six to eight at 6-month intervals after course five; courses nine and ten were each administered 1 year after the previous course. This means that the entire treatment phase lasted nearly 5 years (57 months). During each course MP (500 mg, diluted in $500 \mathrm{ml}$ of $0.9 \% \mathrm{NaCl}$ solution) was intravenously administered on 5 successive days. On day 3, mitoxantrone in a dose of $10 \mathrm{mg} / \mathrm{m}^{2}$ body surface was slowly infused (over at least $1 \mathrm{~h}$ ) after the MP. The antiemetic ondansetron $(4 \mathrm{mg}$ ) was given $30 \mathrm{~min}$ before mitoxantrone.

Patients with contraindications to MP (e.g. gastric ulcer, osteoporosis, psychiatric disorders, glaucoma, ongoing infections, severe diabetes mellitus, or severe hypertension) received mitoxantrone as a single-agent therapy. Those patients who developed severe side effects due to MP (e.g. severe hyperglycemia, hypertensive crisis, psychosis) during the study were given mitoxantrone without MP at the following treatment courses.
The white blood cell and platelet counts were determined before and 1,2, and 4 weeks after each treatment. The mitoxantrone dose was reduced to $8 \mathrm{mg} / \mathrm{m}^{2}$ when the white blood cell count fell below $1,000 / \mu 1$ or the platelet count, below $25,000 / \mu 1$ after the previous treatment course. The treatment was postponed when the patient presented with signs of infection.

\section{Exclusion and Dropout Criteria}

Criteria for exclusion were defined as follows: (1) the patient had received a current or previous treatment with mitoxantrone, radiation, other cytotoxic drugs, or (2) had contraindications to mitoxantrone (e.g. CHF, LV-EF $<50 \%$, severe liver and kidney disease, pregnancy).

Criteria for dropout were defined as follows: (1) the patient was not compliant, i.e. did not appear at follow-up treatment, and (2) mitoxantrone caused severe side effects (e.g. serious infections after mitoxantrone therapy; signs of $\mathrm{CHF}$, decrease of the LV-EF to a value $<50 \%$ or by $10 \%$ or more from baseline; new onset of liver or kidney disease, cancer or leukemia [22]; allergy to mitoxantrone).

\section{Cardiac Function Diagnostics}

The following investigations were performed before each treatment course. A transthoracic echocardiogram was done either in the hospital at the Department of Cardiology or in a few cases, outside the hospital by a cardiologist. Standard two-dimensional echocardiography using a Toshiba PowerVision 6000 (Toshiba Europe, Neuss, Germany) system was applied to evaluate cardiac function. LV diameters, i.e. end-diastolic diameter (EDD) and end-systolic diameter (ESD), were measured in the parasternal short axis view to calculate LV fractional shortening (FS) and LV-EF. LV-EF was calculated using the formula derived by Teichholz: $\mathrm{LV}-\mathrm{EF}=\{[7 /(2,4$ LV-EDD)] LV-EDD ${ }^{3}$ - [7/(2,4 LV-ESD)] LV-ESD $\left.{ }^{3}\right\} /\{[7 /(2,4$ LVEDD)] LV-EDD $\left.{ }^{3}\right\}^{2}$ [23], which had been shown to allow calculation of LV volumes with a very good correlation to volumes derived by single-plane angiography [24]. Grossly asymmetrical LV shapes that would limit the value of this formula were not encountered in our study population. Regional wall motion abnormalities (hypokinesia, akinesia, dyskinesia) were assessed in a parasternal short and long axis and in apical 2- and 4-chamber views. A twelve-lead standard ECG was obtained, and the blood pressure was measured in each patient before each treatment course.

\section{Statistical Analysis}

A repeated measures ANOVA with two categorical predictors (gender and progression), treatment cycles 1-9, and LV-EF, EDD and ESD as variables was performed. Post-hoc analysis was done using the Bonferroni method to correct for multiple testing (Statistica 6, StatSoft, Tulsa, Okla., USA).

\section{Results}

Seventy-three patients ( 41 females, 32 males; age 48 \pm 12 years, range $20-75$ years) who received at least four courses of mitoxantrone therapy were included. Twentyfive patients had a PP, 47 patients an SP and 1 patient an RR form of MS. Three of the 73 patients were exclud- 
Table 1. Mean $\pm \mathrm{SD}$ of the EDD, ESD (in $\mathrm{mm}), \mathrm{FS}, \mathrm{EF}$ (in \%) for the first, fourth, sixth and eighth course of mitoxantrone treatment

\begin{tabular}{llllll}
\hline & $\mathrm{n}$ & EDD & ESD & FS & EF \\
\hline Course 1 & 70 & $47.5 \pm 5.7$ & $28.2 \pm 4.7$ & $40.5 \pm 4.2$ & $64.7 \pm 5.7$ \\
& & $35.0-60.0$ & $18.0-39.2$ & $30.0-47.0$ & $51.0-84.0$ \\
\hline Course 4 & 70 & $46.8 \pm 4.5$ & $27.9 \pm 3.7$ & $40.3 \pm 4.4$ & $64.3 \pm 5.9$ \\
& & $38.0-55.0$ & $18.0-36.0$ & $30.0-54.0$ & $51.0-84.0$ \\
\hline Course 6 & 42 & $47.1 \pm 4.3$ & $28.6 \pm 2.9$ & $39.2 \pm 3.8$ & $62.8 \pm 4.7$ \\
& & $39.0-55.0$ & $22.0-35.0$ & $30.0-46.0$ & $50.0-70.0$ \\
\hline Course 8 & 10 & $45.5 \pm 3.3$ & $27.1 \pm 4.6$ & $38.3 \pm 4.0$ & $64.0 \pm 8.1$ \\
& & $39.0-52.0$ & $22.0-35.0$ & $30.0-44.0$ & $51.0-74.0$
\end{tabular}

There was no significant difference over the treatment courses. ed during the study (2 patients discontinued therapy; 1 patient with a previous history of ischemic heart disease developed atrial fibrillation after the second treatment with mitoxantrone). Of the remaining 70 patients, 63 were given a combined therapy with mitoxantrone and MP; 7 patients received only mitoxantrone for the following reasons: 1 patient had suffered from a necrosis of the femoral head prior to therapy; 1 patient had a history of a gastric ulcer and gastrointestinal bleeding before therapy; 1 patient with diabetes mellitus had previously developed severe hyperglycemia during treatments with MP; 1 patient had a psychosis in his medical history; 2 patients refused MP treatment; and 1 patient developed a transient manic disorder during the second mitoxantrone and MP course, and was therefore treated with only mitoxantrone at the following courses. The mean followup time was 23.4 months (range 10-57 months). Nineteen patients received $8-10$ courses, 8 patients 7 courses, 15 patients 6 courses, 13 patients 5 courses and 15 patients 4 courses. The mean cumulative dose of mitoxantrone was $114.0 \pm 33.8 \mathrm{mg}$ (range 56.8-190.0 mg). A total of 371 echocardiograms was performed. Thirty-six echocardiograms were not available for different reasons.

At the beginning of the study, the PP-MS group had a mean EDSS of $5.2 \pm 1.3$ (range 2.5-7.5) points and the SP-MS patients, a mean EDSS of $5.5 \pm 1.0$ (range 3.07.0) points. Our interim analysis after a study period of 5 years revealed that overall, the EDSS remained almost unchanged after four and eight courses in the PP-MS and SP-MS groups, respectively. Patients with PP-MS had a mean EDSS of $5.2 \pm 1.5$ (range 2.5-7.0) points after four courses and $5.1 \pm 1.4$ (range 3.5-6.5) points after eight courses. The SP-MS group had a mean EDSS of $5.4 \pm$
$1.1(3.0-7.0)$ points after four and $5.6 \pm 1.5$ (range 3.08.0) points after eight courses.

Meanwhile, the clinical data on the effects of mitoxantrone and MP on the progression of PP-MS and SP-MS of a subgroup of these patients have been published [25].

All parameters under investigation (EDD, ESD, FS and EF) before courses 1, 4, 6 and 8 are shown in table 1 . Statistical analysis revealed significantly greater values for EDD and ESD in males than in females, whereas no significant difference was detected for the LV-EF. Overall, there was no significant change in any of the echocardiographic parameters over the whole treatment period $[F(14,224)=1.175, p=0.295]$. We did not find any gender-specific difference in the follow-up of cardiac function. Figure 1 shows the LV-EF before therapy and the development during the treatment from course one to nine in males and females. Similarly, the single analysis for each individual patient did not give evidence of deterioration of cardiac function. None of the patients developed any regional wall motion abnormalities or experienced CHF.

\section{Discussion}

\section{Inclusion Criteria and Study Population}

Mitoxantrone was shown to have a highly therapeutic effect in RR-MS, SP-MS [1, 26, 27] and acute progressive MS [2], including mainly young patients with a low EDSS. On the basis of these findings, mitoxantrone was approved by the Food and Drug Administration as a second-line treatment (after the failure of immunomodulatory therapies) for patients with worsening RR-MS or SP- 
Fig. 1. LV-EF during treatment courses one to nine in female (O) and male ( $\square$ ) patients.

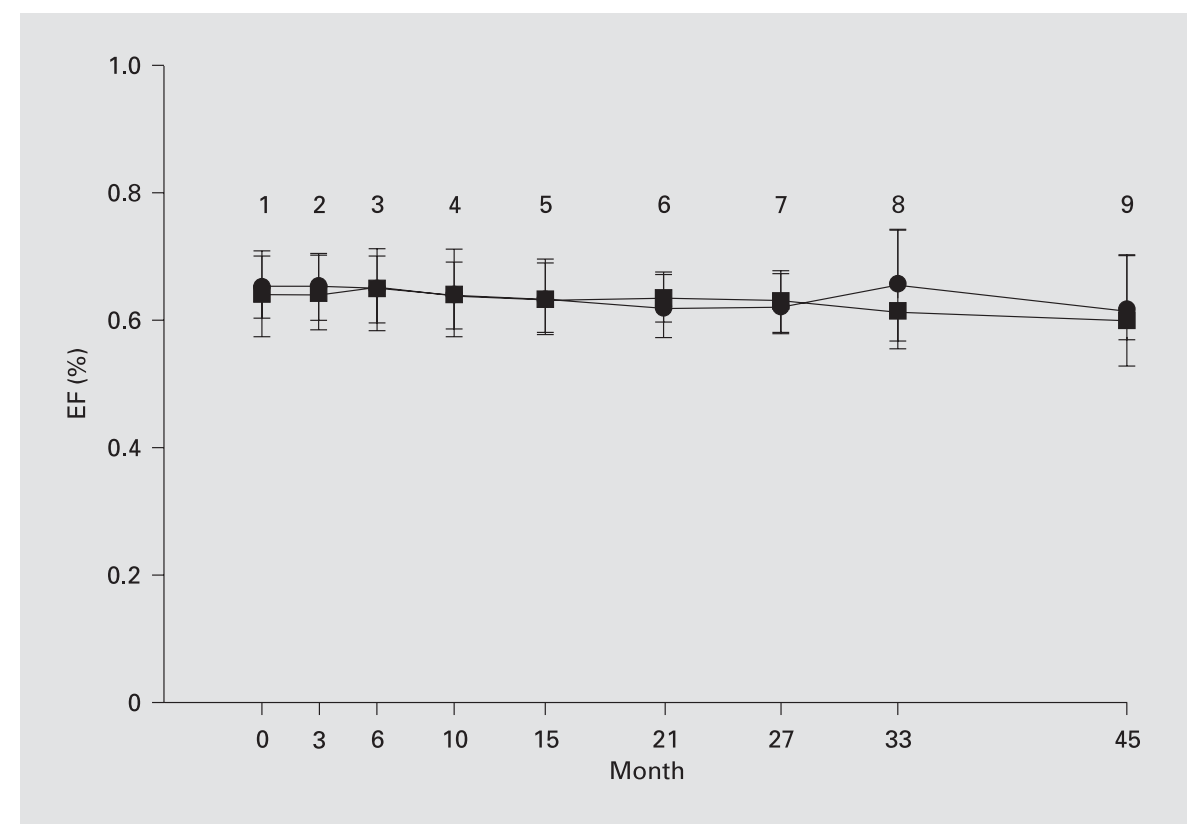

MS. We therefore changed the EDSS inclusion criterium to at least 0.5 points within the preceding 12 months, since we assumed that mitoxantrone might be highly effective in the early phase of the patient's disease and in those patients with low disability. Our assumption was recently supported by the MIMS study $[1,26]$. We decided to also include patients with PP-MS as well as SPMS and RR-MS, although there are currently no approved drugs for the treatment of PP-MS. The effects of several immunosuppressive drugs (such as methotrexate, azathioprine, or cyclophosphamide), which have been tested in progressive MS, are still uncertain and need confirmation by large controlled studies [28, 29]. Before we started the trial, single PP-MS patients had been treated with mitoxantrone in our Department, and it had prevented further progression in most of them. On the basis of these observations, we designed the study to compare treatment effects of mitoxantrone in patients with PP-MS and SP-MS.

\section{Treatment Regimen}

We developed our study design according to that of Edan et al. [2], who had reported in 1997 on the effective, and probably synergistic, combination of mitoxantrone and corticosteroids for treatment of MS, and before the results of the MIMS study were published in 2002 [1]. Edan et al. [2] showed that a monthly administered, combined mitoxantrone $(20 \mathrm{mg})$ and MP therapy $(1 \mathrm{~g})$ over
6 months was superior to a single-agent MP treatment in patients with very active MS. Even now, it is still not known to what extent the treatment effects of mitoxantrone are sustained after stopping therapy. That is why our study included an induction phase, in which mitoxantrone was administered every 3 months, and a maintenance phase, in which the intervals between treatment were increased. This regimen improves the benefit/risk ratio, in particular for patients with only moderate progression of the disease, because the potential cumulative cardiotoxic side effects of mitoxantrone are reached at a late time point during the disease. The treatment protocol called for maximally ten courses of mitoxantrone (10 mg/ $\mathrm{m}^{2}$ body surface), i.e. a total dosage of $100 \mathrm{mg} / \mathrm{m}^{2}$ body surface was not exceeded. Moreover, the cardiotoxicity of anthracyclines is related more to the peak of plasma concentration than to the total systemic exposure or the tissue concentration over time. Thus, in our study mitoxantrone was administered as a slow infusion (over $1 \mathrm{~h}$ ).

\section{Treatment Effect of Mitoxantrone in PP-MS and $S P-M S$}

In the SP-MS group, the combination of mitoxantrone $\left(10 \mathrm{mg} / \mathrm{m}^{2}\right)$ with MP led to a cessation of the progressive disease course in most of our patients or to an improvement in some of them [25]. This result agrees with the data of the multicenter phase III trial [1], in which a significantly greater beneficial effect was shown in patients 
treated with mitoxantrone $12 \mathrm{mg} / \mathrm{m}^{2}$ compared with those who were assigned to mitoxantrone $5 \mathrm{mg} / \mathrm{m}^{2}$ or placebo. In our trial a smaller dosage of mitoxantrone $\left(10 \mathrm{mg} / \mathrm{m}^{2}\right)$ had a similarly beneficial effect. This observation might prove to be of benefit to patients undergoing long-term treatment with mitoxantrone, because the dose-related potential side effects could possibly be reduced. In our trial, approximately one-third of all enrolled patients suffered from PP-MS. Although PP-MS is generally associated with a poorer prognosis than SP-MS, our analysis revealed that these patients also benefit from a combined mitoxantrone $\left(10 \mathrm{mg} / \mathrm{m}^{2}\right)$ and MP therapy like the patients with SP-MS. However, it must be noted that in accordance with the lower incidence of PP-MS, the number of PP-MS patients included is still small.

\section{Potential Cardiotoxicity of Mitoxantrone}

This interim analysis showed no significant changes of the determined parameters (EDD, ESD, FS, EF) over time. Therefore, a mean cumulative dose of about $115 \mathrm{mg}$ mitoxantrone (range 56.8-190.1 mg) did not cause a deterioration of cardiac function after a mean follow-up time of about 2 years (range 10-57 months). None of our patients experienced CHF. The only abnormal cardiac event observed was the occurrence of atrial fibrillation after the second treatment course with mitoxantrone. The patient, however, was 75 years old and had a previous history of ischemic heart disease and hypertension. This might support observations made in earlier studies of a higher cardiotoxicity of mitoxantrone in patients with concomitant (cardiac) diseases [9-12]. These studies showed that only a minor percentage of all treated patients, $0.2 \%$ [11] up to $1.1 \%$ [10], had cardiac side effects. The two main risk factors for cardiotoxicity were concomitant cardiovascular diseases and a higher cumulative dose of mitoxantrone (besides a combined therapy with other chemotherapy or irradiation in cancer patients). Gender appears to be an additive risk factor, since a twofold cardiovascular mortality was found in females after coronary artery bypass grafting [30, 31]. This might be dependent on gender-specific gene expression [32]. In our study population, mitoxantrone was well tolerated and no signs of gender-specific effects of mitoxantrone on cardiac function were observed.

The mean follow-up time in our study was comparable to a recent meta-analysis by Ghalie et al. [33]. In their meta-analysis of 1,378 patients, the safety of mitoxantrone applied as a single-agent therapy for MS was evaluated from three clinical trials, two of them retrospective chart reviews (the MIMS-study by Hartung et al. [1]; a
French study by Edan et al. [14], and a German study by Mauch et al. $[15,16])$. Signs of CHF occurred in only 2 patients, even though 17 of the 779 patients $(2.18 \%)$ with baseline and follow-up evaluation of LV function developed an asymptomatic deterioration of cardiac function with reduction of the LV-EF to $<50 \%$. Asymptomatic LV-EF reduction tended to be more prevalent when higher cumulative doses of $\geq 100 \mathrm{mg} / \mathrm{m}^{2}$ mitoxantrone were applied, but the difference did not reach significance $(p=0.06)$. Limitations of this meta-analysis were the different treatment regimens and protocols to evaluate $\mathrm{LV}$ function. Echocardiograms were performed in only $57 \%$ of all enrolled patients. The intervals between cardiac testing were greater than in our study: in the MIMS study [1] echocardiograms were performed before the initiation of therapy and on follow-up at 12, 24 and 36 months; in the French multicenter study [14], echocardiograms were obtained before therapy and after 6, 24 and 60 months.

Overall, the risk of cardiotoxicity due to mitoxantrone cannot be ruled out. Especially the long-term safety of mitoxantrone needs to be investigated, since Goffette et al. [34] recently reported that three MS patients developed a delayed CHF 24, 29 and 60 months after receiving the last dose of mitoxantrone. However, two of them had received cyclophosphamide prior to the mitoxantrone therapy; this could be an additional risk factor for a mitoxantrone-induced cardiotoxicity.

We intend to continue recruiting new patients for our ongoing prospective study and to extend the mean followup time to a total of 5 years.

\section{Conclusion}

This interim analysis suggests that repeated evaluation of LV function at each successive treatment course with mitoxantrone may not be necessary in low-risk patients without signs of previous cardiac disease, because the probability of cardiotoxicity due to mitoxantrone is very low in these patients. However, the inclusion of more patients and the prolongation of follow-up duration with higher cumulative doses of mitoxantrone will be necessary to substantiate this conclusion.

\section{Acknowledgements}

We thank Ms. Judy Benson for copy-editing the manuscript. 


\section{References}

1 Hartung HP, Gonsette R, Konig N, Kwiecinski H, Guseo A, Morrissey SP, et al: Mitoxantrone in progressive multiple sclerosis: a placebo-controlled, double-blind, randomised, multicentre trial. Lancet 2002;360:20182025.

2 Edan G, Miller D, Clanet M, Confavreux C, Lyon-Caen O, Lubetzki C: Therapeutic effect of mitoxantrone combined with methylprednisolone in multiple sclerosis: a randomized multicentre study of active disease using MRI and clinical criteria. J Neurol Neurosurg Psychiatry 1997;62:112-118.

3 Noseworthy JH, Hopkins MB, Vandervoort MK, Karlik SJ, Lee DH, Penman M, et al: An open trial evaluation of mitoxantrone in the treatment of progressive MS. Neurology 1993; 43:1401-1406.

4 Gonsette RE, Demonty L: Immunosuppression with mitoxantrone in multiple sclerosis: a pilot study for 2 years in 22 patients. Neurology 1990;40(suppl 1):261.

5 Kappos L, Gold R, Künstler E, et al: Mitoxantrone in the treatment of rapidly progressive MS: A pilot study with serial gadolinium-enhanced MRI (abstract). Neurology 1990; 40(suppl 1):261.

6 Goodin DS, Arnason BG, Coyle PK, Frohman EM, Paty DW: The use of mitoxantrone (novantrone) for the treatment of multiple sclerosis. Report of the therapeutics and technology assessment subcommittee of the American Academy of Neurology. Neurology 2003;61:13321338.

7 Avasarala JR, Cross AH, Clifford DB, Singer BA, Siegel BA, Abbey EE: Rapid onset mitoxantrone-induced cardiotoxicity in secondary progressive multiple sclerosis. Mult Scler 2003; 9:59-62.

8 Herman EH, Zhang J, Hasinoff BB, Clark JR, Ferrans VJ: Comparison of the structural changes induced by doxorubicin and mitoxantrone in the heart, kidney and intestine and characterization of the $\mathrm{Fe}(\mathrm{III})$-mitoxantrone complex. J Mol Cell Cardiol 1997;29:24152430.

9 Dukart G, Barone JS: An overview of cardiac episodes following mitoxantrone administration. Cancer Treat Symp 1984;3:35-41.

10 Foster BJ, Lev K, Bergemann C, et al: Cardiac advents in Phase II trials with mitoxantrone. Cancer Treat Symp 1984;3:43-46.

11 Posner LE, Dukart G, Goldberg J, Bernstein T, Cartwright K: Mitoxantrone: an overview of safety and toxicity. Invest New Drugs 1985;3: 123-132.
12 Mather FJ, Simon RM, Clark GM, Von Hoff DD: Cardiotoxicity in patients treated with mitoxantrone: Southwest Oncology Group Phase II studies. Cancer Treat Rep 1987;71: 609-613.

13 Hartung HP, Gonsette R, Morrissey S, Krapf H, Fazekas F: Mitoxanrone; in Rudick RA, Goodkin DE (eds): Multiple Sclerosis Therapeutics. London, Martin Dunitz 1999, pp 335 348.

14 Edan G, Brochet B, Clanet M, et al: Safety profile of mitoxantrone in a cohort of 800 multiple sclerosis patients. Mult Scler 2001;7(suppl 1): S14.

15 Mauch E, Eisenmann S, Hahn A, et al: Mitoxantrone (MITOX) in the treatment of patients with multiple sclerosis (MS): A large single center experience (abstract). American Neurological Association, 1999.

16 Mauch E, Kornhuber HH, Krapf H, Fetzer U, Laufen H: Treatment of multiple sclerosis with mitoxantrone. Eur Arch Psychiatry Clin Neurosci 1992;242:96-102.

17 De Castro S, Cartoni D, Millefiorini E, Funaro S, Gasperini C, Morino S, et al: Noninvasive assessment of mitoxantrone in cardiotoxicity in relapsing-remitting multiple sclerosis. J Clin Pharmacol 1995;35:627-632.

18 Strotmann JM, Spindler M, Weilbach FX, Gold R, Ertl G, Voelker W: Myocardial function in patients with multiple sclerosis treated with low-dose mitoxantrone. Am J Cardiol 2002;89:1222-1225.

19 Poser CM, Paty DW, Scheinberg L, McDonald WI, Davis FA, Ebers GC, et al: New diagnostic criteria for multiple sclerosis: Guidelines for research protocols. Ann Neurol 1983;13:227231.

20 McDonald WI, Compston A, Edan G, Goodkin D, Hartung HP, Lublin FD, et al: Recommended diagnostic criteria for multiple sclerosis: Guidelines from the International Panel on the diagnosis of multiple sclerosis. Ann Neurol 2001;50:121-127.

21 Kurtzke JF: Rating neurological impairment in multiple sclerosis: An expanded disability status scale (EDSS). Neurology 1983;33:14441452.

22 Voltz R, Starck M, Zingler VC, Strupp M: Mitoxantrone therapy in multiple sclerosis and acute leukaemia: a case report out of 644 treated patients. Mult Scler 2004;10:472-474.
23 Teichholz LE, Kreulen T, Herman MV, Gorlin $\mathrm{R}$ : Problems in echocardiographic volume determinations: echocardiographic-angiographic correlations in the presence of absence of asynergy. Am J Cardiol 1976;37:7-11.

24 Kronik G, Slany J, Mosslacher H: Comparative value of eight M-mode echocardiographic formulas for determining left ventricular stroke volume. A correlative study with thermodilution and left ventricular single-plane cineangiography. Circulation 1979;60:1308-1316.

25 Zingler VC, Strupp M, Jahn K, Gross A, Hohlfeld R, Brandt T: The effect of combined mitoxantrone and methylprednisolone therapy in primary and secondary progressive multiple sclerosis. Nervenarzt 2005;76:740-747.

26 Gonsette RE: Mitoxantrone in progressive multiple sclerosis: when and how to treat? J Neurol Sci 2003;206:203-208.

27 Millefiorini E, Gasperini C, Pozzilli C, et al: Randomized placebo-controlled trial of mitoxantrone in relapsing-remitting multiple sclerosis: 24-month clinical and MRI outcome. J Neurol 1997;244:153-159.

28 Zephir H, de Seze J, Duhamel A, et al: Treatment of progressive forms of multiple sclerosis by cyclophosphamide: a cohort study of 490 patients. J Neurol Sci 2004;218:73-77.

29 Yudkin PL, Ellison GW, Ghezzi A, et al: Overview of azathioprine therapy in multiple sclerosis. Lancet 1993;338:1051-1055.

30 Athanasiou T, Al-Ruzzeh S, Del Stanbridge R, Casula RP, Glenville BE, Amrani M: Is the female gender an independent predictor of adverse outcome after off-pump coronary artery bypass grafting? Ann Thorac Surg 2003;75: 1153-1160.

31 Koch CG, Mangano CM, Schwann N, Vaccarino $\mathrm{V}$ : Is it gender, methodology, or something else? J Thorac Cardiovasc Surg 2003; 126:932935.

32 Ruel M, Bianchi C, Khan TA, Xu S, Liddicoat JR, Voisine P, et al: Gene expression profile after cardiopulmonary bypass and cardioplegic arrest. J Thorac Cardiovasc Surg 2003; 126 : 1521-1530.

33 Ghalie RG, Edan G, Laurent M, Mauch E, Eisenmann S, Hartung HP, et al: Cardiac adverse effects associated with mitoxantrone (novantrone) therapy in patients with MS. Neurology 2002;59:909-913.

34 Goffette S, van Pesch V, Sindic CJM: Severe delayed heart failure in three MS patients previously treated with mitoxantrone. Mult Scler 2004; 10(suppl 2):S269. 